\title{
Effect of thalidomide with melphalan and prednisone on health-related quality of life (HRQoL) in elderly patients with newly diagnosed multiple myeloma: a prospective analysis in a randomized trial
}

\author{
Silvia G. R. Verelst $\cdot$ F. Termorshuizen •
}

C. A. Uyl-de Groot • M. R. Schaafsma •

\author{
A. H. M. Ammerlaan • S. Wittebol • H. A. M. Sinnige • \\ S. Zweegman • M. van Marwijk Kooy • \\ R. van der Griend $\cdot$ H. M. Lokhorst $\cdot$ P. Sonneveld • \\ P. W. Wijermans • \\ Dutch-Belgium Hemato-Oncology Cooperative Group \\ (HOVON)
}

Received: 27 September 2010 / Accepted: 22 March 2011 / Published online: 7 April 2011

(C) Springer-Verlag 2011

\begin{abstract}
Thalidomide with melphalan/prednisone (MPT) was defined as standard treatment in elderly patients with multiple myeloma (MM) based on five randomized trials. In one of these trials, HOVON49, a prospective health-related quality-of-life (HRQoL) study was initiated in order to assess the impact of thalidomide on QoL. Patients aged $>65$ years with newly diagnosed $\mathrm{MM}$ were randomized to receive melphalan plus prednisone (MP) or MPT, followed by
\end{abstract}

\footnotetext{
S. G. R. Verelst $(\bowtie) \cdot$ P. Sonneveld

Department of Hematology, Erasmus Medical Center Rotterdam, Rm L407 Rotterdam, PO box 2040, 3000 CA Rotterdam, The Netherlands

e-mail: s.verelst@erasmusmc.nl
}

F. Termorshuizen $\cdot$ A. H. M. Ammerlaan

Department of Trials and Statistics,

Erasmus Medical Center Rotterdam,

Rotterdam, The Netherlands

\section{A. Uyl-de Groot}

Institute for Medical Technology Assessment,

Erasmus University Rotterdam,

Rotterdam, The Netherlands

\section{R. Schaafsma}

Department Internal Medicine, Medical Spectrum Twente, Enschede, The Netherlands

\section{S. Wittebol}

Department of Internal Medicine, Meander Medical Center,

Amersfoort, The Netherlands thalidomide maintenance in the MPT arm. Two hundred eighty-four patients were included in this side study (MP, $n=$ 149; MPT $n=135$ ). HRQoL was assessed with the EORTC Core QoL Questionnaire (QLQ-C30) and the myelomaspecific module (QLQ-MY24) at baseline and at predetermined intervals during treatment. The QLQ-C30 subscales physical function $(P=0.044)$ and constipation $(P<0.001)$ showed an improvement during induction in favour of the

\section{H. A. M. Sinnige}

Department of Internal Medicine, Jeroen Bosch Hospital,

's-Hertogenbosch, The Netherlands

S. Zweegman

Department of Hematology,

VU University Medical Center Amsterdam,

Amsterdam, The Netherlands

M. van Marwijk Kooy

Department of Internal Medicine, Isala Kliniek,

Zwolle, The Netherlands

R. van der Griend

Department of Internal Medicine, Diakonessenhuis Utrecht,

Utrecht, The Netherlands

H. M. Lokhorst

Department of Hematology, University Medical Center Utrecht, Utrecht, The Netherlands

P. W. Wijermans

Department of Hematology, Haga Hospital,

The Hague, The Netherlands 
MP arm. During thalidomide maintenance, the scores for the QLQ-MY24 paraesthesia became significantly higher in the MPT arm $(P<0.001)$. The QLQ-C30 subscales pain $(P=$ $0.12)$, insomnia $(P=0.068)$, appetite loss $(P=0.074)$ and the QLQ-MY24 item sick $(P=0.086)$ scored marginally better during thalidomide maintenance. The overall QoL-scale QLQ-C30-HRQoL showed a significant time trend towards more favourable mean values during protocol treatment without differences between MP and MPT. For the QLQC30 subscales emotional function and future perspectives, difference in favour of the MPT arm from the start of treatment was observed ( $P=0.018$ and $P=0.045$, respectively) with no significant 'time $\times$ arm' interaction, indicating a persistent better patient perspective with MPT treatment. This study shows that the higher frequency of toxicity associated with MPT does not translate into a negative effect on HRQoL and that MPT holds a better patient perspective.

Keywords Randomized clinical trial - Multiple myeloma . Thalidomide · Elderly Quality of life

\section{Introduction}

Multiple myeloma (MM), a neoplasm of plasma cells, affects 6 per 100,000 individuals each year worldwide [1, 2]. It is a progressive disease marked by increased risk of infections, skeletal destruction (leading to bone pain and fractures), renal failure and anemia [3]. Since the 1960s melphalan plus prednisone (MP) was the standard treatment for elderly patients ( $>65$ years) with MM. However, the response duration was rather short and the median survival about 3 years. Recently, five randomized clinical phase III trials in which thalidomide was added to the melphalan/prednisone schedule as first line treatment demonstrated a higher response rate, a qualitative better response, increased progression-free survival and in three out of five also a benefit in overall survival [4-9]. Melphalan plus prednisone and thalidomide (MPT) is now regarded as a new standard for treatment of multiple myeloma in the elderly patient. However, in all these trials, thalidomide was associated with a higher incidence of adverse effects, such as deep-vein thrombosis, peripheral neuropathy, constipation and infections (CTC 3/4). In contrast, the better treatment efficacy leads to a reduction of MM-related symptoms. It is therefore imperative to clarify to what extent both aspects, increased efficacy and higher risk of adverse events, affect the quality of life of patients. In the HOVON 49 trial comparing MP vs. MPT followed by thalidomide maintenance in the MPT arm, a prospective health-related quality-of-life (HRQoL) study was integrated with the purpose of evaluating the impact of thalidomide on patients' quality of life [9]. Here, we report the results of this assessment.

\section{Methods}

Study design and treatment

Details on the HOVON 49 study were recently published. In summary, the HOVON 49 was a prospective phase III randomized (1:1), open-label, multicenter trial that compared standard melphalan plus prednisone with melphalan plus prednisone and thalidomide for patients aged $>65$ years with newly diagnosed symptomatic multiple myeloma. Patients were included who had symptomatic MM, stage Ib, II or III according to the Salmon and Durie criteria [10]. Treatment consisted of 8 cycles of melphalan $0.25 \mathrm{~kg} / \mathrm{kg}$ per day and prednisone $1 \mathrm{mg} / \mathrm{kg}$ per day for 5 days. Cycles were given every 4 weeks. In the MPT arm, thalidomide $200 \mathrm{mg} /$ daily was added. In case of disease progression or non-response, patients should stop protocol treatment and might start second-line treatment. Patients were allowed to receive more than eight courses in case of ongoing response until a plateau phase of $\mathrm{M}$ protein was achieved. Patients randomized to the MPT arm and who reached the plateau level of response received maintenance therapy of thalidomide $50 \mathrm{mg} /$ daily until progression or relapse.

During this trial treatment with bisphosphonates was recommended, either pamidronate or clodronate. At the start of this study, there were no guidelines on use of thrombosis prophylaxis. Low molecular weight heparin was recommended as standard during MPT treatment from 2005 on, and during maintenance, low-dose aspirin was advised $[9,11]$. The study was conducted according to the Declaration of Helsinki and the guidelines of good clinical practice. The trial was registered as ISRCTN90692740.

\section{Patients}

In principle, the HRQoL study included all clinically evaluable patients who participated in the HOVON 49 study. For this analysis, those patients were included for whom at least one valid HRQoL assessment was available, either at baseline or during the treatment trajectory.

\section{HRQoL questionnaires}

In this study two validated questionnaires were used: European Organization for Research and Treatment of Cancer (EORTC) QLQ-C30 and the EORTC QLQ-MY24. The QLQ-C30 is a multidimensional, cancer-specific, quality-of-life questionnaire developed by the EORTC Study Group on Quality of Life for use in international clinical trial settings [12]. It includes five functional scales (physical, role, emotional, social and cognitive functioning), three symptom scales (fatigue, pain, and nausea and vomiting), a global health status/quality-of-life scale and a 
number of single items assessing additional symptoms (dyspnoea, sleep disturbance, constipation and diarrhoea) and perceived financial impact. For the majority of the QLQ-C30 items, a four-point Likert-type response scale is used. Exceptions are the items for the global quality-of-life scale (where a seven-point scale is used). The EORTC QLQ-MY24 questionnaire measures specific aspects that are relevant for the quality of life (QoL) in multiple myeloma patients. The questionnaire contains the following scales: pain, side effects of treatment, social support, body image and future perspectives [13].

The patients' individual score of each subscale was calculated as the sum and the mean of the available items for that scale. All subscale responses were linearly converted to $0-100$ scales. For the functional and global quality-of-life scales, a higher score represents a better level of functioning. For the symptom scales and items, a higher score reflects a greater degree of symptomatology. If the number of missing items for a certain scale was less than half of the number of needed items for that scale, the score was calculated on the basis of the available items. Otherwise, the whole scale was coded as missing.

\section{Data collection}

According to the study protocol, QoL should be measured at the following time points:

1. At study entry, prior to the first cycle ('baseline')

2. After cycle 3, approximately at 3 months after the start of cycle 1

3. After cycle 8 , approximately at 9 months after the start of cycle 1

4. At 12 months after start cycle 1

5. At 18 months after start cycle 1

After obtaining informed consent, the baseline questionnaire was handed out by the QoL coordinator of the participating hospital. The subsequent questionnaires were sent to the patient by the HOVON data centre with a prestamped reply envelope to facilitate returning. If the patient refused to transmit his/her address to the HOVON data centre, all questionnaires were handed out and returned by the QoL coordinator. The QoL coordinator received a reminder email in time in order to hand out the questionnaire at the correct time point.

Statistical analysis

\section{Definition of discrete treatment trajectory time points}

Variation between patients in the number of received cycles of melphalan/prednisone was present. The 3-month, 8-month and 12-month questionnaires as indicated in the study protocol may represent the induction phase, end of treatment or late follow-up according to the individual patient his/her unique treatment trajectory. In order to keep the comparison between the two randomization arms unconfounded by the actual phase of the treatment protocol, the time points of QoL assessment were categorized in four broad classes: at study entry before the start of the first cycle or within 3 days thereafter (baseline ' $t 0$ '), during or shortly after (within a week) the phase of induction treatment (induction 'i.'), during the period following the first week after induction treatment until the start of the next treatment off protocol (post-induction 'p.'), and after progression, relapse or starting a next treatment off protocol (re-treatment 'r.').

To include a maximum number of returned questionnaires in the analysis, the dates of filling out the questionnaires were categorized according to the number of months after the start of cycle 1 for the induction and post-induction phases separately. For induction (i.) these time points were: 'i. months $1-2$ ', 'i. month 3', 'i. months 4-7' and 'i. $\geq 8$ months'. For post-induction these time points were: 'i. $<12$ months' (prior to the indicated ranges below in case of early stopping of induction treatment), 'i. 12 months' (range 10.8-15.8) and 'i. 18 months' (range 15.8-21.3). No subdivision in time points was made for those questionnaires that were filled out after relapse, progression or starting a new treatment off protocol (r.).

\section{Statistical model}

To obtain estimates of the mean scores for the EORTC QLQ-C30 and QLQ-MY24 subscales that might be regarded as continuously distributed outcome variables at each of the above described discrete treatment trajectory time points, a linear mixed model was used with a random intercept for patient effects and time according to the protocol trajectory (categorical), arm of randomization and their interaction as fixed effects. The subscales that were based on a single item, and thus, had a multinomial distribution, were likewise analyzed using (ordinal) logistic regression with a random intercept.

The estimated effect of arm of randomization without taking the interaction between treatment trajectory time and arm into account is indicative of the difference between the two arms already present at baseline and, hence, should be zero in case of perfect randomization. The estimated interaction effects between treatment trajectory time and arm of randomization are indicative of the effect of arm in addition to the (possible) difference by arm already present at baseline and, hence, might be assigned to the additional treatment with thalidomide in the experimental arm. Tests were performed using the Wald's test for different linear combinations of estimated parameters. The mean of a 
continuously EORTC QLQ-C30 or QLQ-MY24 subscale for a certain treatment trajectory time point and arm of randomization was calculated as the sum of the intercept, the estimated effect parameter for arm of randomization, the appropriate effect parameter for treatment trajectory time point and the appropriate effect parameter for interaction between time and arm of randomization. The estimated proportion of patients with an answer in a certain category of a mono-item subscale (i.e. a multinomial distributed subscale) was likewise calculated by arm of randomization and treatment trajectory time point on the basis of the estimated effect parameters.

The results of a selection of subscale were graphically displayed together with the $P$ values and degrees of freedom of the Wald's tests.

Next to time, arm and terms for interaction between time and arm, last registered response $(\geq \mathrm{PR}$ vs. $<\mathrm{PR}$ ) prior to a certain HRQoL assessment and toxicity as registered during the same time interval of the treatment trajectory were included in the multivariate model. Toxicity was defined as any dose reduction or discontinuation of melphalan, prednisone or thalidomide during induction or maintenance due to side effects. In this way, it was assessed whether response and toxicity were associated with the HRQoL, either in favourable or unfavourable sense, and whether the above described results on time, arm and interaction between both were modified when these factors were included in the multivariate model.

The data of all patients with at least one HRQoL assessments, either at baseline, during treatment or during follow-up, were included in the analysis. Random-intercept models make it possible to include the data of patients with missing data as well. Those patients with only one QoL assessment contribute to the location of the intercept and to differences by randomization arm.

All analyses were performed with STATA statistical software, version 10.1 (StataCorp., TX, USA).

\section{Results}

\section{Patient characteristics}

The HOVON 49 MM trial accrued 344 patients from 58 centres between 2002 and 2007. Median follow-up at the time of last contact was 3.25 years. Of the 333 eligible patients, 284 participated in the prospective HRQoL side study, of whom 149 were randomized to receive MP and 135 MPT. No significant differences in clinical characteristics were present between participants and non-participants. The clinical characteristics of participating patients are shown by arm of randomization in Table 1.

At baseline, the mean EORTC scores were comparable between MP and MPT, except for emotional function, future perspectives, social support and global health and QoL subscale ( $P=0.018, P=0.045, P=0.032$ and $P=0.050$ respectively), which were in favour of MPT (Table 2).

Patient functioning by treatment trajectory time point and arm of randomization

\section{Continuously distributed subscales}

For the overall QLQ-C30 global health status subscale (QLQC30-HR-QOL), a significant time trend towards highermore favourable - mean values during the protocol treatment trajectory was observed but no differences between MP and MPT could be established [Fig. 1 (1.1)]. No significant 'time $\times$ arm' interaction effects were observed from induction to post-induction and after progression/relapse indicating that thalidomide had no effect on this subscale.

For the QLQ-C30 physical function subscale [Fig. 1 (1.2)], role function and emotional functioning [Fig. 1 (1.3)] statistically significant and favourable time trends during protocol treatment were found as well $(P<0.001)$. For the QLQ-C30 subscale physical function, a treatmentrelated improvement in favour of the MP arm was found early during induction phase, yielding a significant 'time $\times$ arm' interaction effect during induction $(P=0.044)$. During post-induction and after starting a new treatment, no significant differences by arm of randomization were observed anymore.

For the QLQ-C30 emotional function subscale [Fig. 1 (1.3)] as well as for the QLQ-MY24 future perspective subscale [Fig. 1 (1.6)], a difference in favour of the MPT arm during the complete treatment trajectory was observed. However, these differences were already present at baseline ( $P=0.018$ and $P=0.045$, respectively) and, hence, no significant 'time $\times$ arm' interaction could be established, indicating a persistent better patient perspective with MPT treatment.

For the QLQ-MY24 side effects subscale no differences between the two arms, neither at baseline, nor during protocol treatment and during follow-up was found. After looking into detail of the different items of this MY24 subscale, however, highly significant terms for interaction during post-induction ('p.') and after progression/relapse or starting a new treatment ('r.') could be established for the item paraesthesia in favour of the MP arm [Fig. 1 (1.8)]. No differences between the two arms were found at baseline and during early induction $(P=0.69$ and $P=0.66$, respectively) but after 3 months, higher scores were found in the MPT arm which became significant during post-induction and after progression/relapse or starting a new treatment off protocol $(P<0.001)$.

For the item sick, (borderline) significant terms for interaction during post-induction and after progression/ 
Table 1 Baseline characteristics QoL participation MP arm vs. MPT arm

\begin{tabular}{|c|c|c|c|c|c|c|}
\hline \multirow[b]{2}{*}{ Total } & \multicolumn{2}{|c|}{ MP (no. and percentage) } & \multicolumn{2}{|c|}{ MPT (no. and percentage) } & \multicolumn{2}{|c|}{ Total (no. and percentage) } \\
\hline & 149 & $100 \%$ & 135 & $100 \%$ & 284 & $100 \%$ \\
\hline Sex & \multicolumn{6}{|c|}{$\left(P=0.641, \chi^{2}\right)$} \\
\hline M & 82 & $55 \%$ & 78 & $58 \%$ & 160 & $56 \%$ \\
\hline $\mathrm{F}$ & 67 & $45 \%$ & 57 & $42 \%$ & 124 & $44 \%$ \\
\hline Age & \multicolumn{6}{|c|}{$(P=0.345, t$ test $)$} \\
\hline Median & \multicolumn{2}{|c|}{$72(65-84)$} & \multicolumn{2}{|c|}{$71(65-83)$} & \multicolumn{2}{|c|}{$72(65-84)$} \\
\hline Age in three classes & \multicolumn{6}{|c|}{$\left(P=0.249, \chi^{2}\right)$} \\
\hline$\leq 70$ & 52 & $35 \%$ & 60 & $44 \%$ & 112 & $39 \%$ \\
\hline $71-75$ & 50 & $34 \%$ & 37 & $27 \%$ & 87 & $31 \%$ \\
\hline$>75$ & 47 & $32 \%$ & 38 & $28 \%$ & 85 & $30 \%$ \\
\hline WHO status & \multicolumn{6}{|c|}{$\left(P=0.101, \chi^{2}\right)$} \\
\hline WHO 0 & 45 & $30 \%$ & 59 & $44 \%$ & 104 & $37 \%$ \\
\hline WHO 1 & 76 & $51 \%$ & 58 & $43 \%$ & 134 & $47 \%$ \\
\hline WHO 2 & 21 & $14 \%$ & 15 & $11 \%$ & 36 & $13 \%$ \\
\hline WHO 3 & 7 & $5 \%$ & 3 & $2 \%$ & 10 & $4 \%$ \\
\hline Stage of disease (Salmon \& Durie (S\&D)) & \multicolumn{6}{|c|}{$(P=0.809$, exact $)$} \\
\hline 1B & \multicolumn{2}{|l|}{0} & 1 & $1 \%$ & 1 & $0 \%$ \\
\hline $2 \mathrm{~A}$ & 39 & $26 \%$ & 32 & $24 \%$ & 71 & $25 \%$ \\
\hline $2 \mathrm{~B}$ & 1 & $1 \%$ & 2 & $1 \%$ & 3 & $1 \%$ \\
\hline $3 \mathrm{~A}$ & 97 & $65 \%$ & 87 & $64 \%$ & 184 & $65 \%$ \\
\hline $3 \mathrm{~B}$ & 12 & $8 \%$ & 13 & $10 \%$ & 25 & $9 \%$ \\
\hline International Staging System (ISS) (calculated) & \multicolumn{6}{|c|}{$(P=0.454$, exact $)$} \\
\hline Stage I & 36 & $24 \%$ & 37 & $27 \%$ & 73 & $26 \%$ \\
\hline Stage II & 35 & $23 \%$ & 37 & $27 \%$ & 72 & $25 \%$ \\
\hline Stage III & 25 & $17 \%$ & 25 & $19 \%$ & 50 & $18 \%$ \\
\hline Unknown & 53 & $36 \%$ & 36 & $27 \%$ & 89 & $31 \%$ \\
\hline M-protein heavy chain & \multicolumn{6}{|c|}{$(P=0.839$, exact $)$} \\
\hline $\operatorname{Ig} \mathrm{A}$ & 43 & $24 \%$ & 39 & $29 \%$ & 82 & $29 \%$ \\
\hline $\operatorname{IgG}$ & 92 & $62 \%$ & 80 & $59 \%$ & 172 & $61 \%$ \\
\hline $\operatorname{IgD}$ & 0 & & 1 & $1 \%$ & 1 & $0 \%$ \\
\hline LCD & 14 & $9 \%$ & 15 & $11 \%$ & 29 & $10 \%$ \\
\hline M-protein light chain & \multicolumn{6}{|c|}{$(P=0.322$, exact $)$} \\
\hline Kappa & 99 & $66 \%$ & 82 & $61 \%$ & 181 & $64 \%$ \\
\hline Lambda & 49 & $33 \%$ & 53 & $39 \%$ & 102 & $36 \%$ \\
\hline Unknown & 1 & $1 \%$ & 0 & & 1 & $0 \%$ \\
\hline Number of skeletal lesions & \multicolumn{6}{|c|}{$(P=0.846$, exact $)$} \\
\hline None & 41 & $28 \%$ & 39 & $29 \%$ & 80 & $28 \%$ \\
\hline Single & 8 & $5 \%$ & 7 & $5 \%$ & 15 & $5 \%$ \\
\hline 2 lesions & 9 & $6 \%$ & 4 & $3 \%$ & 13 & $5 \%$ \\
\hline $3+$ lesions & 88 & $59 \%$ & 77 & $57 \%$ & 165 & $58 \%$ \\
\hline Unknown & 3 & $2 \%$ & 8 & $5 \%$ & 11 & $3 \%$ \\
\hline
\end{tabular}

relapse or starting a new treatment in favour of the MPT arm were found $[P=0.086$ and $P=0.027$, respectively; Fig. 1 (1.7)].

For the other items of the dimension side effects, no differences in favour of either the MP or MPT arm were found.
For the QLQ-MY24 social support subscale, significant differences between the two arms were present, both at baseline $(P=0.032)$ and during the protocol treatment trajectory (induction, $P=0.02$; post-induction, $P=0.024$ ). However, the differences were not unequivocal in favour of either MP or MPT, thus, a closely reasoned argument is 


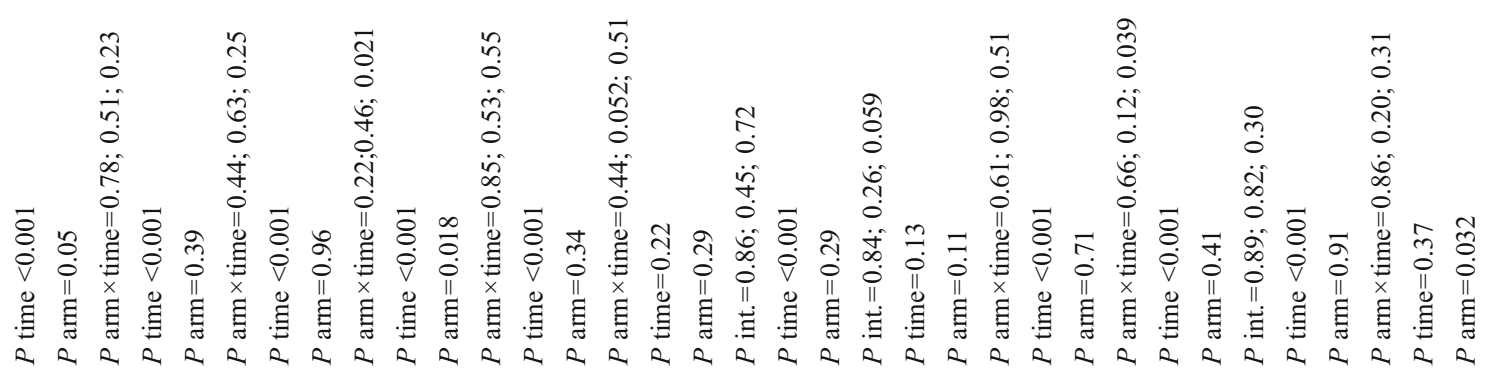

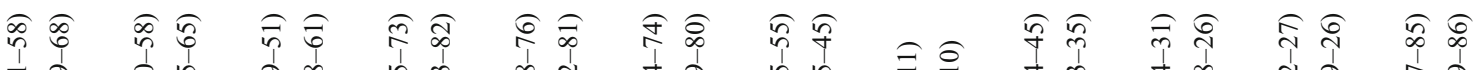

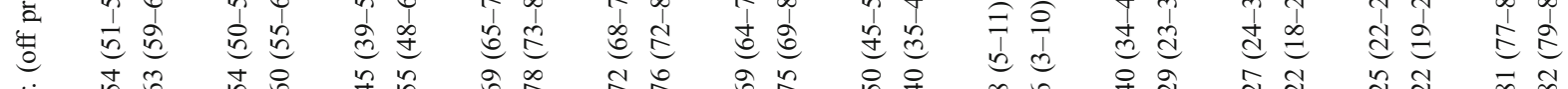

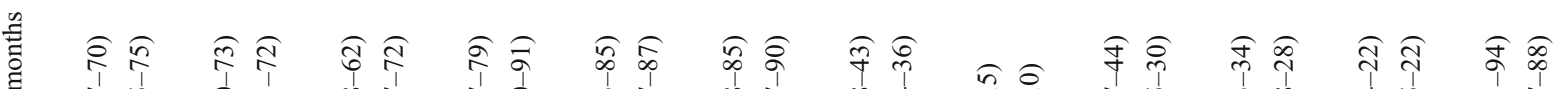

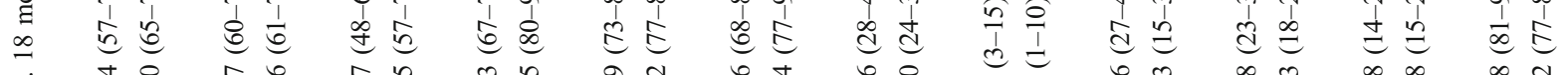

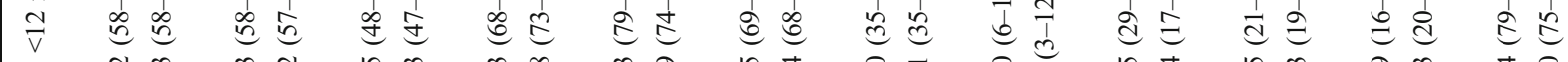
$\dot{a} \quad \delta$

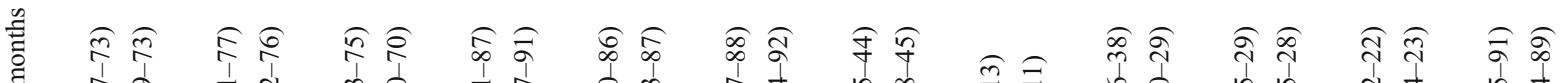

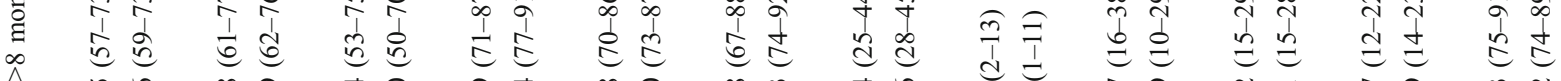

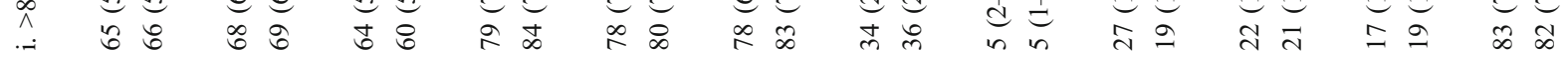

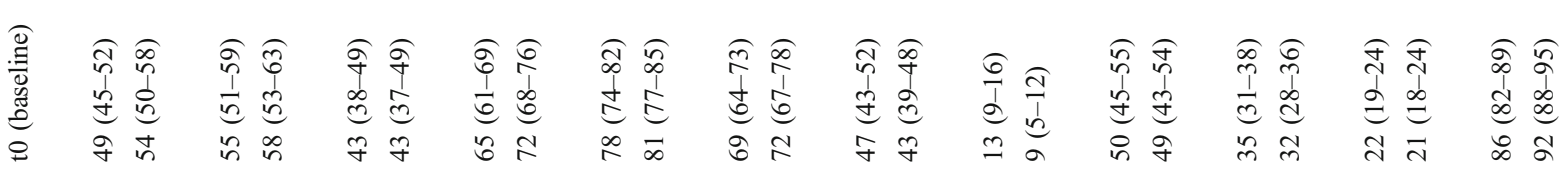

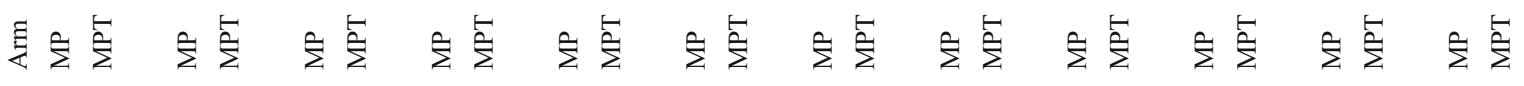




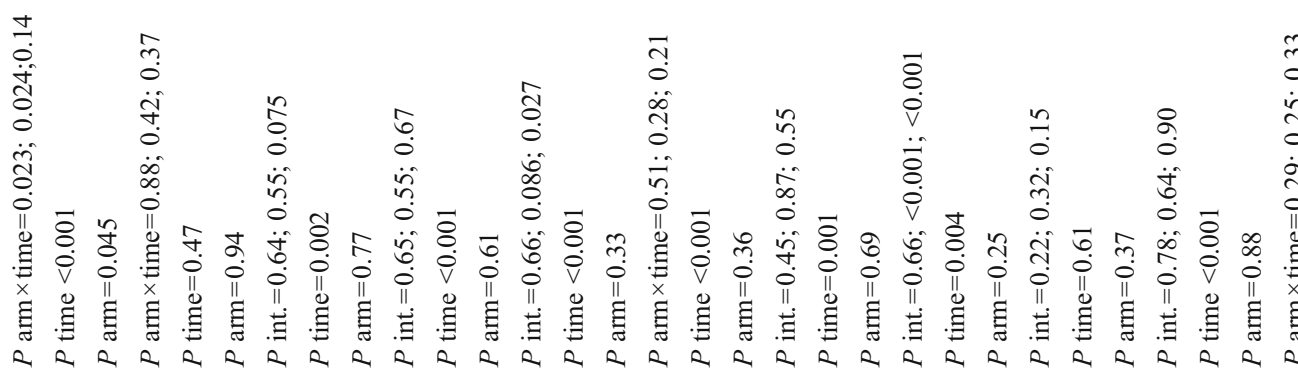

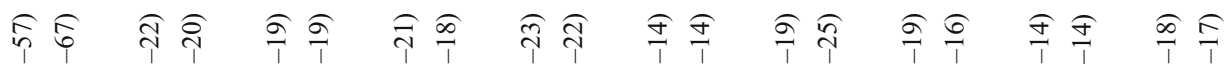

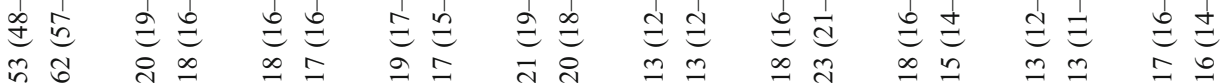

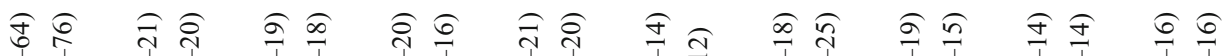

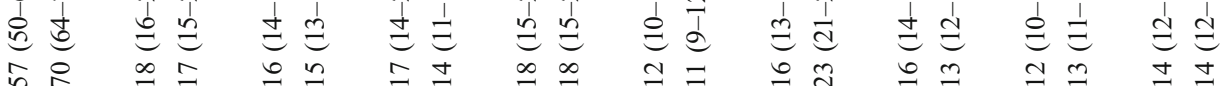

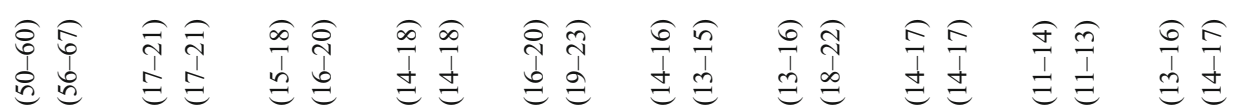

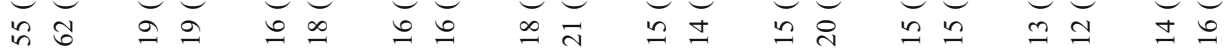

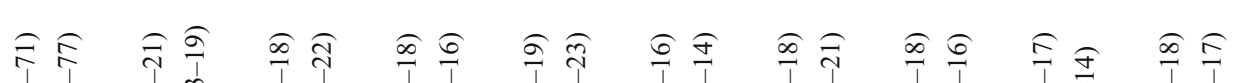

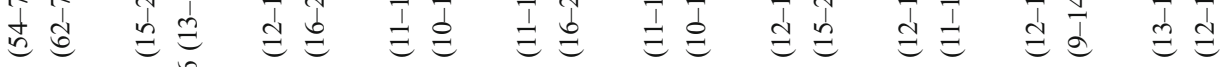

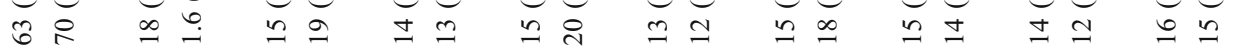

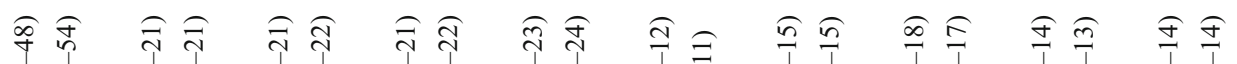

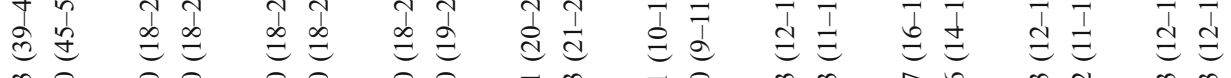

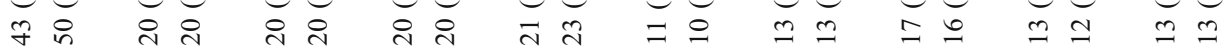

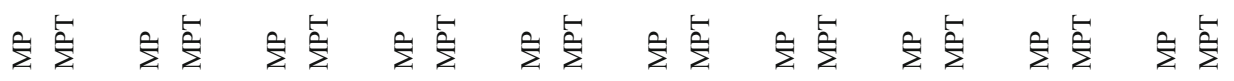

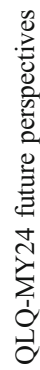

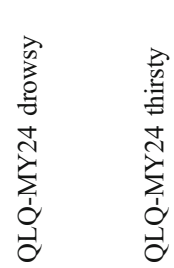

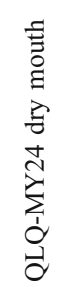

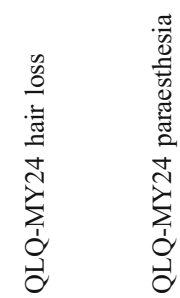

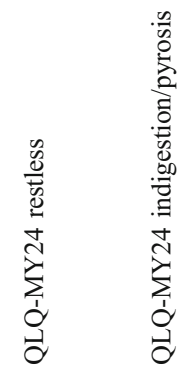

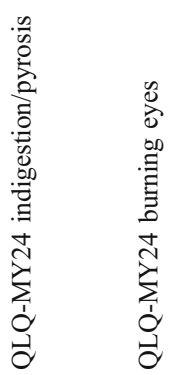

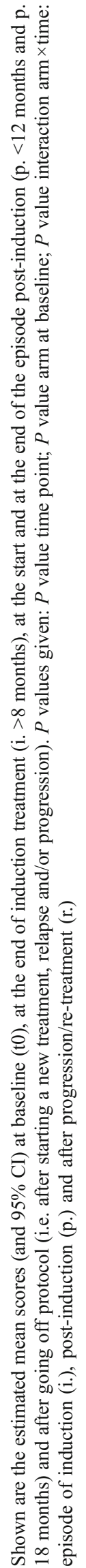


1.1 QLQ-C30 Global health

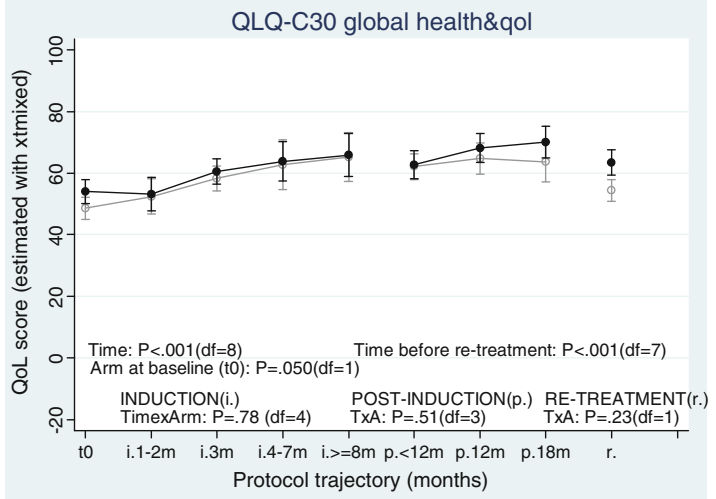

\subsection{QLQ-C30 Emotional function}

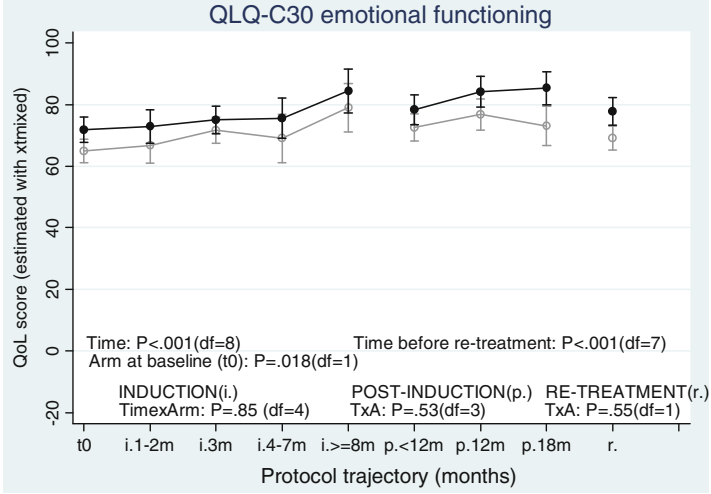

\subsection{QLQ-C30 Pain}

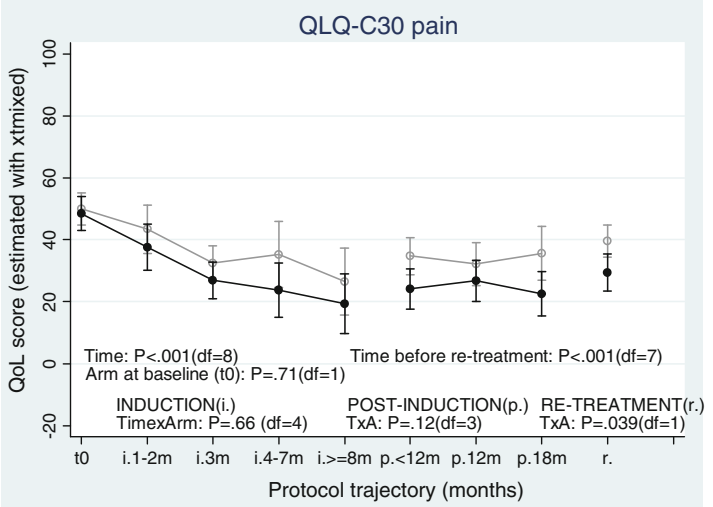

1.7 Sick

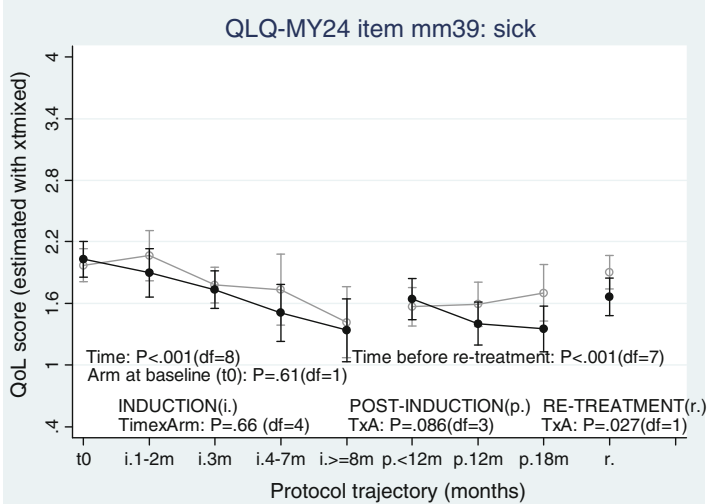

1.2 QLQ-C30 Physical function

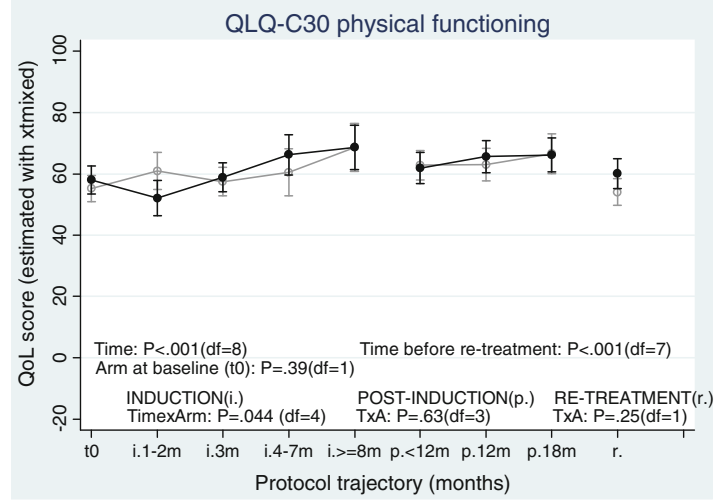

\subsection{QLQ-C30 Fatigue}

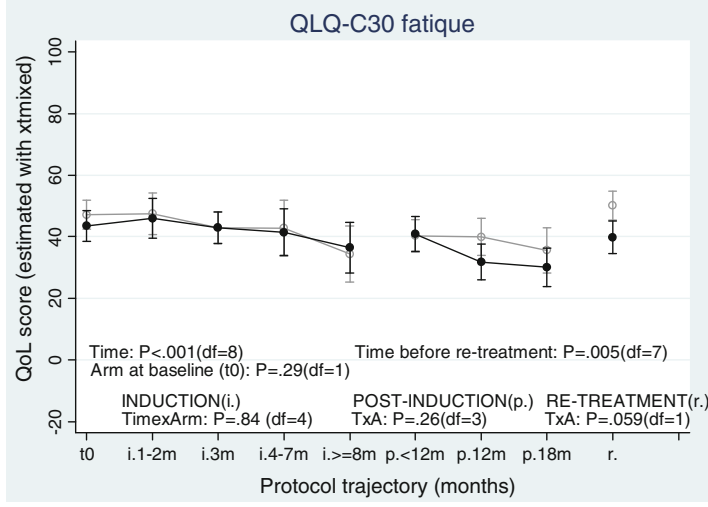

\subsection{QLQ-MY24 Future perspectives}

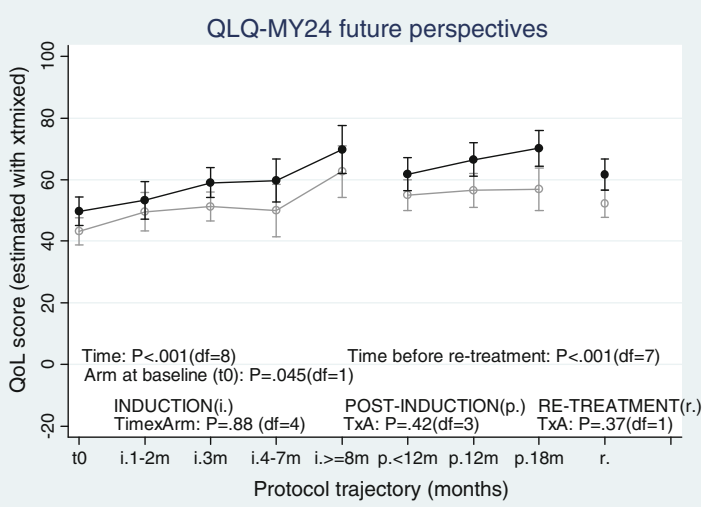

1.8 Paraesthesia

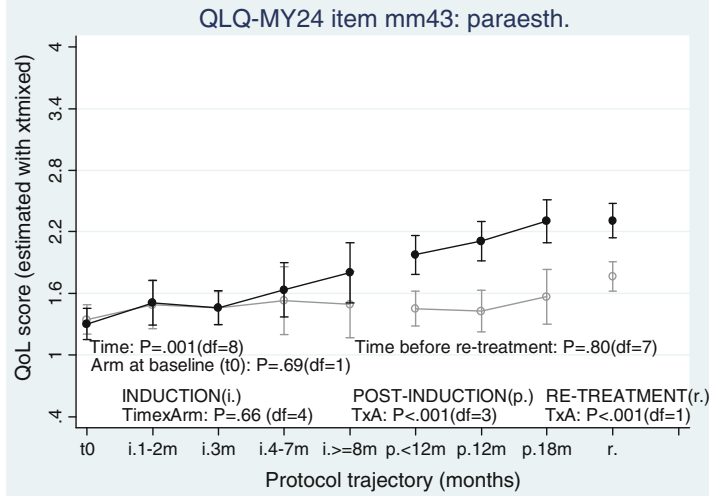


Fig 1 Comparison of QoL parameters during therapy with MP or MPT in patients with multiple myeloma (gray lines MP, black lines $\mathrm{MP}+$ thalidomide). Output from linear mixed model

hard to give. For the QLQ-C30 subscale cognitive function, a borderline significant $(P=0.052)$ interaction between arm and time point during the post-induction phase was found. However, these differences were not consistently in favour of the MP or the MPT arm.

\section{Subscales with multinomial distribution}

In Fig. 2 the estimated proportions of patients with an answer in a certain category of a mono-item subscale are shown by arm of randomization and time point in the treatment trajectory. The proportions for each time point and arm of randomization separately sum up to $100 \%$. For the graphs in the Fig. 2 (2.1-2.3), the answer categories 'Moderate' and 'Severe' were taken together in one category because of small numbers in these separate categories. For the same reason, for the graph in Fig. 2 (2.4, body image), the categories 'No' and 'Mild' were taken together in one category.

\subsection{QLQ-C30 Insomnia}

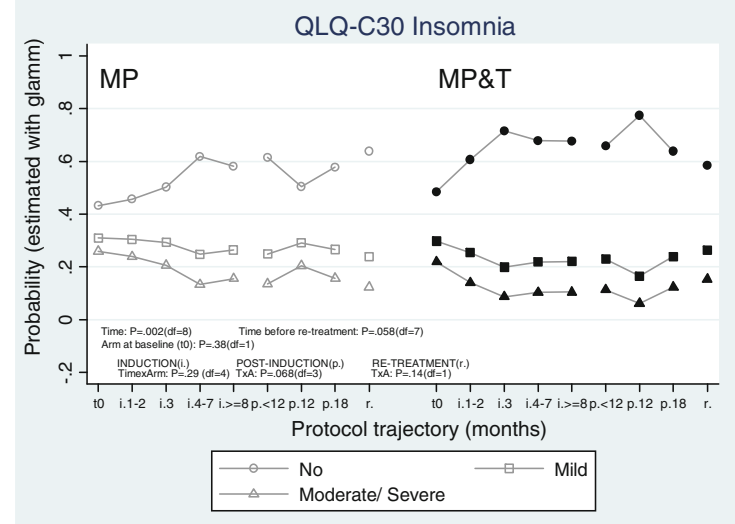

2.3 QLQ-C30 Constipation

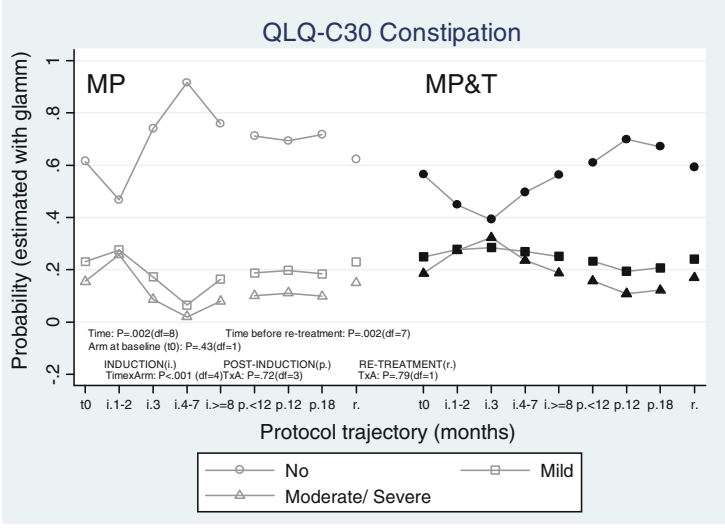

For the QLQ-C30 subscales insomnia [Fig. 2 (2.1)], appetite loss [Fig. 2 (2.2)] and constipation [Fig. 2 (2.3)], a favourable time trend towards higher percentages of patients without these symptoms $(P=0.058, P=0.001$ and $P=0.002$, respectively) was established in both arms. For constipation a significant 'time $\times$ arm' interaction effect $(P<0.001)$ was found during induction, associated with higher percentages of patients with this symptom in the MPT arm. During postinduction, a borderline significant 'time $\times$ arm' interaction was found in favour of the MPT arm for insomnia $(P=0.068)$ and appetite loss $(P=0.074)$, indicating more favourable percentages of patients without this symptom in the MPT arm. For appetite loss this continued after progression/relapse or starting a new treatment $(P=0.006)$.

For the QLQ-MY24 body image subscale [Fig. 2 (2.4)], a significant 'time $\times$ arm' interaction during postinduction $(P=0.009)$ was established associated with an unfavourable time trend towards a higher percentage of patients in the symptom category 'severe' in the MPT arm. After starting a new treatment, no difference in percentages between the two arms was found $(P=0.36$, Table 3).

\subsection{QLQ-C30 Appetite loss}

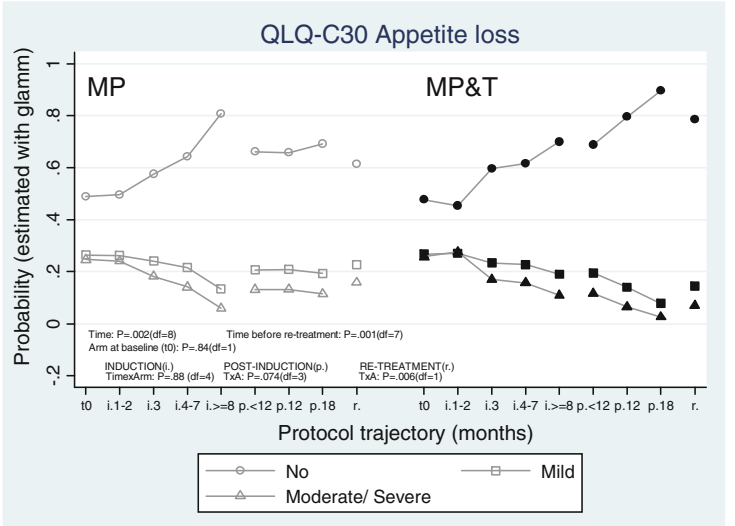

\subsection{QLQ-MY24 Body image}

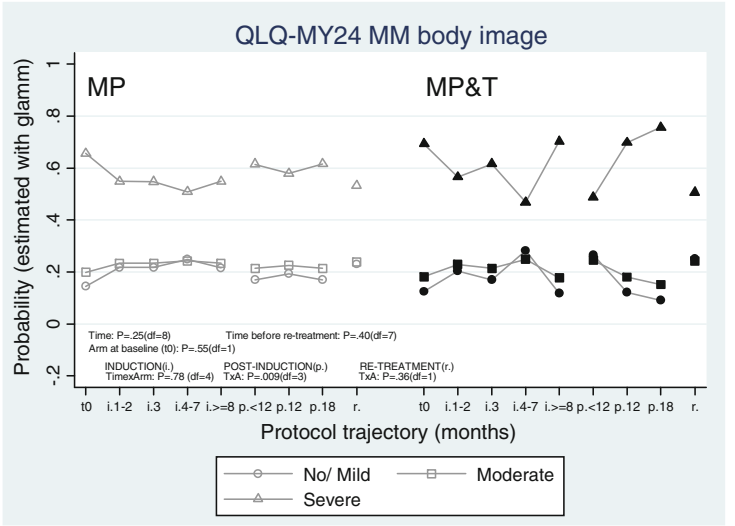

Fig 2 Comparison of QoL parameters during therapy with MP or MPT in patients with multiple myeloma (gray lines MP, black lines MP+thalidomide). Output from ordinal logistic regression 
Table 3 QoL by dimension, arm of randomization (MP vs. MPT) and time point in treatment trajectory

Induction (i.) Post-induction (p.)

After re-treatment/relapse/ progression (r.)

\begin{tabular}{|c|c|c|c|c|c|c|}
\hline \multirow[t]{2}{*}{ QoL dimension } & & t0 (baseline) & i. $>8$ months & p. $<12$ months & p. 18 months & r. (off protocol) \\
\hline & Arm & & & & & \\
\hline \multirow[t]{2}{*}{ QLQ-C30 dyspnoea mild/severe (\%) } & MP & $32.7 / 17.5$ & $35.4 / 25.1$ & $33.4 / 19.0$ & $29.4 / 13.0$ & $35.0 / 23.5$ \\
\hline & MPT & $34.2 / 20.8$ & $30.1 / 13.7$ & $35.6 / 26.4$ & $31.7 / 15.8$ & $34.9 / 22.9$ \\
\hline \multicolumn{2}{|l|}{$\begin{array}{l}P \text { value time point }=0.18(d f=8) \\
P \text { value arm }=0.42(d f=1), P \text { value arm } \times \text { time }=\end{array}$} & $0.11(d f=4)$ & & $0.36(d f=3)$ & & $0.38(d f=1)$ \\
\hline \multirow[t]{2}{*}{ QLQ-C30 insomnia mild/severe (\%) } & MP & $30.8 / 25.9$ & $26.3 / 15.4$ & $24.9 / 13.5$ & $26.5 / 15.7$ & $23.8 / 12.3$ \\
\hline & MPT & $29.7 / 21.8$ & $21.9 / 10.4$ & $22.9 / 11.3$ & $23.8 / 12.3$ & $26.2 / 15.2$ \\
\hline \multicolumn{2}{|l|}{$\begin{array}{l}P \text { value time point }=0.002(d f=8) \\
P \text { value } \operatorname{arm}=0.38(d f=1), P \text { value arm } \times \text { time }=\end{array}$} & $0.29(d f=4)$ & & $0.68(d f=3)$ & & $0.14(d f=1)$ \\
\hline \multirow[t]{2}{*}{ QLQ-C30 appetite loss mild/severe (\%) } & MP & $26.4 / 24.6$ & $13.3 / 6.0$ & $20.7 / 13.1$ & $19.3 / 11.4$ & $22.7 / 15.8$ \\
\hline & MPT & $26.6 / 25.8$ & $18.9 / 11.0$ & 19.5/11.6 & $7.7 / 2.6$ & $14.5 / 6.9$ \\
\hline \multicolumn{2}{|l|}{$\begin{array}{l}P \text { value time point }=0.002(d f=8), \\
P \text { value arm }=0.84(d f=1), P \text { value arm } \times \text { time }=\end{array}$} & $0.88(d f=4)$ & & $0.74(d f=3)$ & & $0.006(d f=1)$ \\
\hline \multirow[t]{2}{*}{ QLQ-C30 constipation mild/severe (\%) } & MP & $23.1 / 15.4$ & $16.2 / 7.9$ & $18.6 / 10.0$ & $18.4 / 9.8$ & $22.8 / 15.0$ \\
\hline & MPT & $24.9 / 18.5$ & $24.9 / 18.6$ & $23.3 / 15.7$ & $20.6 / 12.2$ & $24.0 / 16.8$ \\
\hline \multicolumn{2}{|l|}{$\begin{array}{l}P \text { value time point }=0.002(d f=8) \\
P \text { value arm }=0.43(d f=1), P \text { value arm } \times \text { time }=\end{array}$} & $<0.001(d f=4)$ & & $0.72(d f=3)$ & & $0.79(d f=1)$ \\
\hline \multirow[t]{2}{*}{ QLQ-C30 diarrhoea mild/severe (\%) } & MP & $12.2 / 45.8$ & $14.8 / 6.2$ & $13.7 / 5.4$ & $11.9 / 4.3$ & $16.8 / 7.6$ \\
\hline & MPT & $13.4 / 5.3$ & $8.5 / 2.7$ & $12.1 / 4.5$ & $9.2 / 3.0$ & $14.8 / 6.2$ \\
\hline \multicolumn{2}{|l|}{$\begin{array}{l}P \text { value time point }=0.70(d f=8) \\
P \text { value } \operatorname{arm}=0.70(d f=1), P \text { value arm } \times \text { time }=\end{array}$} & $0.52(d f=4)$ & & $0.77(d f=3)$ & & $0.42(d f=1)$ \\
\hline \multirow[t]{2}{*}{ QLQ-C30 financial problems mild/severe (\%) } & MP & $10.2 / 3.5$ & $16.8 / 8.6$ & $12.5 / 5.2$ & $14.2 / 6.6$ & $16.8 / 8.7$ \\
\hline & MPT & $7.8 / 2.1$ & $10.1 / 3.3$ & $9.0 / 2.7$ & $10.3 / 3.5$ & $12.3 / 4.9$ \\
\hline \multicolumn{2}{|l|}{$\begin{array}{l}P \text { value time point }=0.038(d f=8), \\
\quad P \text { value arm }=0.33(d f=1), P \text { value arm } \times \text { time }=\end{array}$} & $0.39(d f=4)$ & & $0.98(d f=3)$ & & $0.72(d f=1)$ \\
\hline \multirow[t]{2}{*}{ QLQ-C30 body image moderate/severe (\%) } & MP & $19.8 / 65.6$ & $23.4 / 54.9$ & $21.4 / 61.5$ & $21.3 / 61.5$ & $23.8 / 53.1$ \\
\hline & MPT & $18.2 / 69.3$ & $17.7 / 70.3$ & $24.5 / 48.8$ & $15.2 / 75.6$ & $24.3 / 50.6$ \\
\hline \multicolumn{2}{|l|}{$\begin{array}{l}P \text { value time point }=0.25(d f=8) \\
\quad P \text { value } \operatorname{arm}=0.55(d f=1), P \text { value arm } \times \text { time }=\end{array}$} & $0.78(d f=4)$ & & $0.009(d f=3)$ & & $0.36(d f=1)$ \\
\hline
\end{tabular}

Shown are the estimated percentages at baseline (t0), at the end of induction treatment (i. $>8$ months), at the start and at the end of the episode post-induction (p. $<12$ months and p. 18 months) and after going off protocol (i.e. after starting a new treatment, relapse and/or progression). $P$ values given: $P$ value time point; $P$ value arm at baseline; $P$ value interaction arm $\times$ time: episode of induction (i.), post-induction (p.) and after progression $/$ re-treatment (r.)

Inclusion of response and toxicity data in the multivariate models

\section{Continuously distributed subscales}

A favourable response (at least PR) was (borderline) significantly associated with more favourable scores on the QoL-C30 dimensions: global health, physical function, role function, emotional function, social function, fatigue, nausea/vomiting, pain and the MY24-dimensions pain, side effects and future perspectives. The only significant association with toxicity that caused dose reduction or discontinuation was with the dimension role function, and this was in unfavourable direction (i.e. more toxicity gave a lower score for role function).

For a number of these dimensions, the time trend towards more favourable scores disappeared or became less pronounced, e.g. QLQ-C30 dimensions global health, physical function, and emotional function. This finding suggests that the favourable time trend along the treatment trajectory is strongly associated with the appearance of a favourable response on therapy. However, no substantial influence of adjustment for response and toxicity on the effect of arm or on the arm $\times$ time interactions was found, which implies that comparison between the two arms is not confounded by either response or toxicity.

\section{Subscales with multinomial distribution}

Both a favourable response $(\geq P R)$ and the presence of toxicity leading to dose reduction or discontinuation were unfavourably and significantly associated with a higher score on the QLQ-C30 dimension dyspnoea. For the dimensions insomnia and appetite loss, a favourable 
response $(\geq \mathrm{PR})$ appeared to be significantly associated with a more favourable (i.e. lower) score on these dimensions. For these dimensions, the time trend along the treatment trajectory disappeared in terms of statistical significance after adjustment for response and toxicity. As was the case with the continuously distributed subscales, no substantial influence of adjustment for response and toxicity on the effect of arm or on the arm $\times$ time interactions was found, which implies that comparison between the two arms is not confounded by either response or toxicity.

Inclusion of gender and hemoglobin data in the multivariate models

Similar results were found when effects where adjusted for gender and hemoglobin at baseline.

For both gender and hemoglobin at baseline, no influence of adjustment on the effect of arm or on the arm $\times$ time interactions was found.

When looking at the different QoL dimensions, we observed, on average, that the QoL scores for females were more unfavourable compared to males. For example, for females, an unfavourable higher mean score was found for the scales nausea/vomiting ( $\beta$-coefficient $5.37 ; P=0.001$ ), appetite loss $(\beta$-coefficient $2.06 ; P=0.020)$, as well as for specific side effects of myeloma treatment $(\beta$-coefficient 3.80; $P=0.019)$. Females also reported unfavourable lower scores for physical functioning $(\beta$-coefficient $-7.87 ; P=$ 0.003 ), emotional functioning ( $\beta$-coefficient $-4.74 ; P=$ $0.039)$, social functioning $(\beta$-coefficient $-6.51 ; P=0.018)$ and future perspectives ( $\beta$-coefficient $-6.06 ; P=0.026)$, but for all these items, inclusion of gender in the model did not modify the arm effect nor the interaction arm $\times$ time effects.

\section{Discussion}

Combining clinical outcomes of a treatment with assessment of the impact of this treatment on HRQoL provides additional value for evaluating treatment effects [14]. Systematic incorporation of HRQoL measures into clinical trials allows for a comparison of treatment arms that includes the patients' perspective. A review study of Kvam et al. in 2009 showed that there are few randomized clinical trials in MM that include HRQoL as a study end-point [15]. HOVON 49 is, to our knowledge, the first phase III randomized multicenter trial comparing the efficacy of MP vs. MPT combined with a HRQoL assessment.

Treatment with MPT not only showed increased efficacy but also higher frequency of toxicity associated with MPT. The main objective of this study was to evaluate the effect of thalidomide on the quality of life for elderly patients with multiple myeloma. Our study shows that treatment resulted in improvement of the scores on overall global health, fatigue, pain, side effects, isomnia and appetite loss in both the MP and the MPT arm. For the subscales pain, insomnia and appetite loss, differences even in favour of the MPT arm were observed.

However, MPT was associated with a significant increase in paraesthesia from post-induction onwards. Increase of these symptoms after 8 months of treatment is consistent with a cumulative dose-dependent effect of thalidomide [9, 16]. This underscores the need for dose reductions and limited periods of maintenance therapy [16, 17]. In spite of the higher incidence of some toxicities with MPT compared to MP (that is constipation and paraesthesia), this is not reflected in the overall quality-of-life scale QLQ-C30-HRQoL. No unfavourable overall difference in self-reported side effects (QLQ-MY24) between the two arms was observed during the study protocol either. The mean score on the item about feeling sick became even significantly lower in the MPT arm compared to the MP arm during post-induction.

\section{Clinical significance}

A prospective study by Kvam et al. determined the clinical significance of changes in EORTC QLQ-C30 scores in patients with $\mathrm{MM}$ by identifying the smallest difference that matters: the so-called minimally important difference. They concluded that a difference of 6-17 points represents a meaningful change in improvement or deterioration in patients and that only score differences equally or greater than this should be used for treatment recommendations [18]. When applying this definition to our results, it can be concluded that a clinical significant difference was observed for the subscales global health, role functioning, emotional functioning, social functioning, fatigue and pain at the end of post-induction ( $P=18$ months) in favour of the MPT-treated patients compared to MP.

An important strength of this study is the use of a prospective design. We were able to assess the effect of thalidomide on the QoL at different time points during treatment trajectory allowing to differentiate according to stage of treatment. Another strength of our study is the use of a statistical model for repeated measurements, as was also employed in a study on QoL among patients with endometrial cancer [19]. This model makes it possible to include all QoL assessments, as the correlation between measurements from the same patient is taken into account, and the data of patients with missing data on QoL assessments contribute to the estimations as well. This model makes it possible to make a clear distinction between differences between the two arms of randomization already present at baseline and those that may have been caused by the additional treatment with thalidomide after starting the 
induction treatment by inclusion of terms for (time $\times$ arm) interaction. An example is the differences observed between the two randomization arms with respect to QoL scores for the dimensions emotional functioning and future perspective. Both of these differences were already present at baseline and no significant (time $\times$ arm) interaction could be established. Given the random assignment of the treatment arms and the fact that the two groups were well balanced for clinical characteristics, these differences may indicate a persistent better patient perspective with MPT treatment. Since the first questionnaire was filled out prior to treatment but after randomization, we hypothesise that the awareness of being treated with a medication that holds out a prospect of better treatment outcome may in itself be associated with improved feeling of well-being and hope for the future. Another explanation for these findings may be found in the so-called Hawthorne effect. Patients perform better when they know they are under observation for an intervention. Patients receiving the experimental (MPT) treatment might feel more special and therefore score better on both subscales from baseline on forward than patients receiving the standard treatment.

The study has also some limitations. First, not all patients included in the HOVON 49 trial participated and the most burdened patients are the ones least likely to participate. However, this is inherent to each QoL survey. Secondly, while the patients were evaluated during predetermined time points during induction and post-induction treatment, not all patients filled out the questionnaire at the exact same time. This problem was overcome by grouping the results into well-defined phases. Finally, the results do not take into account all potential comorbidities of the patients enrolled in this study. As the QoL assessments were part of a RCT, this does not jeopardise the primary conclusion of the present. There may be different effects of treatment with thalidomide on HRQoL in different subgroups defined on the basis of comorbidities. Such a subgroup analysis should be based on clear a priori hypotheses and might require an additional study and data collection.

In conclusion, this prospective study shows that the higher frequency of adverse effects associated with MPT does not translate into a negative effect on the HRQoL as reported by patients and that MPT holds a better patient perspective. This can be interpreted as a favourable result because the current standard treatment of MPT for elderly patients with MM is not only known to have an improved clinical outcome but also no reduction in HRQoL.

Acknowledgments This study was supported by a grant from The Dutch Cancer Society (KWF). Furthermore, we thank the coordinators in the different hospitals and the people at the Hovon Data Centre for the data collection and, last but certainly not least, all the patients who participated and took the time to fill out the questionnaires.

\section{References}

1. Parkin DM, Bray F, Ferlay J, Pisani P (2005) Global cancer statistics, 2002. CA Cancer J Clin 55(2):74-108

2. Kumar SK, Rajkumar SV, Dispenzieri A, Lacy MQ, Hayman SR, Buadi FK, Zeldenrust SR, Dingli D, Russell SJ, Lust JA, Greipp PR, Kyle RA, Gertz MA (2008) Improved survival in multiple myeloma and the impact of novel therapies. Blood 111(5):25162520. doi:10.1182/blood-2007-10-116129

3. Kyle RA, Gertz MA, Witzig TE, Lust JA, Lacy MQ, Dispenzieri A, Fonseca R, Rajkumar SV, Offord JR, Larson DR, Plevak ME, Therneau TM, Greipp PR (2003) Review of 1027 patients with newly diagnosed multiple myeloma. Mayo Clin Proc 78(1):21-33

4. Facon T, Mary JY, Hulin C, Benboubker L, Attal M, Pegourie B, Renaud M, Harousseau JL, Guillerm G, Chaleteix C, Dib M, Voillat L, Maisonneuve H, Troncy J, Dorvaux V, Monconduit M, Martin C, Casassus P, Jaubert J, Jardel H, Doyen C, Kolb B, Anglaret B, Grosbois B, Yakoub-Agha I, Mathiot C, Avet-Loiseau H, Intergroupe Francophone du M (2007) Melphalan and prednisone plus thalidomide versus melphalan and prednisone alone or reduced-intensity autologous stem cell transplantation in elderly patients with multiple myeloma (IFM 99-06): a randomised trial. Lancet 370(9594):12091218. doi:10.1016/S0140-6736(07)61537-2

5. Hulin C, Facon T, Rodon P, Pegourie B, Benboubker L, Doyen C, Dib M, Guillerm G, Salles B, Eschard JP, Lenain P, Casassus P, Azais I, Decaux O, Garderet L, Mathiot C, Fontan J, Lafon I, Virion JM, Moreau P (2009) Efficacy of melphalan and prednisone plus thalidomide in patients older than 75 years with newly diagnosed multiple myeloma: IFM 01/01 trial. J Clin Oncol 27(22):3664-3670. doi:10.1200/JCO.2008.21.0948

6. Palumbo A, Bringhen S, Caravita T, Merla E, Capparella V, Callea V, Cangialosi C, Grasso M, Rossini F, Galli M, Catalano L, Zamagni E, Petrucci MT, De Stefano V, Ceccarelli M, Ambrosini MT, Avonto I, Falco P, Ciccone G, Liberati AM, Musto P, Boccadoro M, Italian Multiple Myeloma Network, GIMEMA (2006) Oral melphalan and prednisone chemotherapy plus thalidomide compared with melphalan and prednisone alone in elderly patients with multiple myeloma: randomised controlled trial. Lancet 367(9513):825-831. doi:10.1016/S0140-6736(06)68338-4

7. Palumbo A, Bringhen S, Liberati AM, Caravita T, Falcone A, Callea V, Montanaro M, Ria R, Capaldi A, Zambello R, Benevolo G, Derudas D, Dore F, Cavallo F, Gay F, Falco P, Ciccone G, Musto P, Cavo M, Boccadoro M (2008) Oral melphalan, prednisone, and thalidomide in elderly patients with multiple myeloma: updated results of a randomized controlled trial. Blood 112(8):3107-3114. doi:10.1182/blood-2008-04-149427

8. Waage A, Gimsing P, Fayers P, Abildgaard N, Ahlberg L, Bjorkstrand B, Carlson K, Dahl IM, Forsberg K, Gulbrandsen N, Haukas E, Hjertner O, Hjorth M, Karlsson T, Knudsen L, Nielsen JL, Linder O, Mellqvist UH, Nesthus I, Rolke J, Strandberg M, Sorbo JH, Wisloff F, Juliusson G, Turesson I (2010) Melphalan and prednisone plus thalidomide or placebo in elderly patients with multiple myeloma. Blood. doi:10.1182/ blood-2009-08-237974

9. Wijermans P, Schaafsma M, Termorshuizen F, Ammerlaan R, Wittebol S, Sinnige H, Zweegman S, van Marwijk Kooy M, van der Griend R, Lokhorst H, Sonneveld P, Dutch-Belgium Cooperative Group HOVON (2010) Phase III study of the value of thalidomide added to melphalan plus prednisone in elderly patients with newly diagnosed multiple myeloma: the HOVON 49 Study. J Clin Oncol 28 (19):3160-3166. doi:10.1200/JCO.2009.26.1610

10. Durie BG, Salmon SE (1975) A clinical staging system for multiple myeloma. Correlation of measured myeloma cell mass with presenting clinical features, response to treatment, and survival. Cancer 36(3):842-854 
11. Minnema MC, Breitkreutz I, Auwerda JJ, van der Holt B, Cremer FW, van Marion AM, Westveer PH, Sonneveld P, Goldschmidt H, Lokhorst HM (2004) Prevention of venous thromboembolism with low molecular-weight heparin in patients with multiple myeloma treated with thalidomide and chemotherapy. Leukemia 18(12):2044-2046. doi:10.1038/sj.leu.2403533

12. Aaronson NK, Ahmedzai S, Bergman B, Bullinger M, Cull A, Duez NJ, Filiberti A, Flechtner H, Fleishman SB, de Haes JC et al (1993) The European Organization for Research and Treatment of Cancer QLQ-C30: a quality-of-life instrument for use in international clinical trials in oncology. J Natl Cancer Inst 85(5):365-376

13. Cocks K, Cohen D, Wisloff F, Sezer O, Lee S, Hippe E, Gimsing P, Turesson I, Hajek R, Smith A, Graham L, Phillips A, Stead M, Velikova G, Brown J, Group EQoL (2007) An international field study of the reliability and validity of a disease-specific questionnaire module (the QLQ-MY20) in assessing the quality of life of patients with multiple myeloma. Eur J Cancer 43 (11):1670-1678. doi:10.1016/j.ejca.2007.04.022

14. Lee SJ, Richardson PG, Sonneveld P, Schuster MW, Irwin D, San Miguel JF, Crawford B, Massaro J, Dhawan R, Gupta S, Anderson $\mathrm{KC}$ (2008) Bortezomib is associated with better health-related quality of life than high-dose dexamethasone in patients with relapsed multiple myeloma: results from the APEX study. Br J Haematol 143 (4):511-519. doi:10.1111/j.1365-2141.2008.07378.x

15. Kvam AK, Fayers P, Hjermstad M, Gulbrandsen N, Wisloff F (2009) Health-related quality of life assessment in randomised controlled trials in multiple myeloma: a critical review of methodology and impact on treatment recommendations. Eur $\mathbf{J}$ Haematol 83(4):279-289. doi:10.1111/j.1600-0609.2009.01303.x

16. Mohty B, El-Cheikh J, Yakoub-Agha I, Moreau P, Harousseau JL, Mohty M (2010) Peripheral neuropathy and new treatments for multiple myeloma: background and practical recommendations. Haematologica 95(2):311-319. doi:10.3324/haematol.2009.012674

17. Palumbo A, Davies F, Kropff M, Blade J, Delforge M, Leal da Costa F, Garcia Sanz R, Schey S, Facon T, Morgan G, Moreau $P$ (2010) Consensus guidelines for the optimal management of adverse events in newly diagnosed, transplant-ineligible patients receiving melphalan and prednisone in combination with thalidomide (MPT) for the treatment of multiple myeloma. Ann Hematol 89(8):803-811. doi:10.1007/s00277-0100925-1

18. Kvam AK, Fayers P, Wisloff F (2009) What changes in healthrelated quality of life matter to multiple myeloma patients? A prospective study. Eur J Haematol. doi:10.1111/j.16000609.2009.01404.x

19. Nout RA, Putter H, Jurgenliemk-Schulz IM, Jobsen JJ, Lutgens LC, van der Steen-Banasik EM, Mens JW, Slot A, Stenfert Kroese MC, van Bunningen BN, Smit VT, Nijman HW, van den Tol PP, Creutzberg CL (2009) Quality of life after pelvic radiotherapy or vaginal brachytherapy for endometrial cancer: first results of the randomized PORTEC-2 trial. J Clin Oncol 27(21):3547-3556. doi:10.1200/JCO.2008.20.2424 